\title{
Reentrance phenomenon in superconductor/ferromagnet nanostructures and their application in superconducting spin valves for superconducting electronics
}

\author{
A.S. Sidorenko \\ D. Ghitsu Institute of Electronic Engineering and Nanotechnologies ASM, MD-2028 Kishinev, Moldova \\ Institut für Physik, Universität Augsburg, D-86159 Augsburg, Germany \\ E-mail: anatoli.sidorenko@kit.edu
}

Received January 2, 2017, published online May 25, 2017

\begin{abstract}
In superconductor/ferromagnet layered structures, a Fulde-Ferrell-Larkin-Ovchinnikov-like inhomogeneous superconducting pairing give rise. The singlet and zero-projection triplet components of the pairing oscillate in space, and the presence of interfaces causes interference phenomena. As the result of the interference, the superconducting critical temperature $T_{c}$ oscillates as a function of the ferromagnetic layer thicknesses or, even more spectacular, reentrant superconductivity appears. Two ferromagnetic layers can be combined with a superconducting layer into a superconducting spin valve. Proper design and choice of the material parameters give possibility to control superconducting $T_{c}$ manipulating with magnetic configurations in the system. The conditions to get large spin-valve effect, i.e., a large shift in the critical temperature, are reviewed in the article.
\end{abstract}

PACS: 74.78.-w Superconducting films and low-dimensional structures;

74.78. Na Mesoscopic and nanoscale systems;

73.50.-h Electronic transport phenomena in thin films;

74.25. $-\mathbf{q}$ Properties of superconductors;

85.25.-j Superconducting devices.

Keywords: superconducting films and multilayers nanostructures, superconducting spin valves, interference phenomena.

\section{Introduction}

Superconductivity (S) and ferromagnetism (F) are antagonistic long-range orders which cannot coexist in a homogeneous material [1] (see, however, reviews on coexistence and interaction of superconductivity and magnetism in Chevrel phases and ternary rhodium borides [2], borocarbides [3] and ruthenates [4]). Heterostructures comprising nanometer-scale layers of superconducting and ferromagnetic layers offer new physical picture of the interaction of superconductivity and magnetism, in which the superconducting pairing function penetrates in the ferromagnetic layers in an oscillating manner. If the oscillation scale and the pairing function decay length are comparable with the ferromagnetic layers thickness, as well as the superconducting layer thickness is comparable with the superconducting coherence length, various interference effects can be expected. In this review we focus on mesoscopic oscillations of the super- conducting transition temperature $T_{c}$ which arise as a result of modulation of the coupling between the superconducting and ferromagnetic layers when varying the ferromagnetic layer thickness. One superconducting layer and two ferromagnetic layers already offer functionality determined by mutual alignment of magnetic moments of the ferromagnetic layers. This functionality can be utilized to build a superconducting spin valve discussed at the end of the article.

\section{Oscillations of superconducting $T_{c}$ in $\mathrm{S} / \mathrm{F}$ bilayers}

In this section we consider plane contact of a superconducting film with a ferromagnetic metal film. At an $\mathrm{S} / \mathrm{F}$ interface the quasi-one-dimensional Fulde-Ferrell-LarkinOvchinnikov (FFLO) like state can be generated in the F material [5-10]. Due to exchange splitting of the conduction band (Fig. 1) the singlet Cooper pairs acquire finite pairing momentum $\hbar Q_{F}=E_{\mathrm{ex}} / v_{F}$ because wave vectors of 


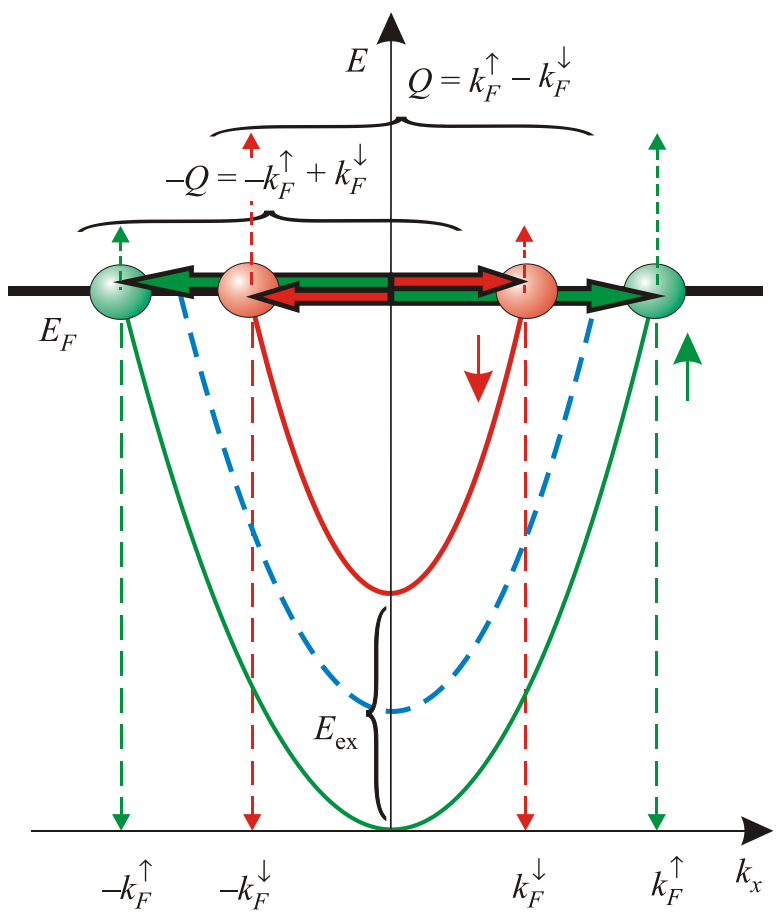

Fig. 1. (Color online) Origin of the FFLO state. The conduction band of a ferromagnetic metal is spin-split $\left(E_{\mathrm{ex}}\right)$ by the exchange field. Paired electrons (red and green balls) from the minority (red) and majority (green) spin subbands (wave vectors are indicated in the respective colours) establish the FFLO-like paired state with finite momentum along the $x$ axis $\hbar Q_{F}=\hbar \Delta k_{F}=E_{\mathrm{ex}} / v_{F}$.

the minority-subband and majority-subband electrons are not equal. Then, the pairing function of this state does not simply decays as it would be in a nonmagnetic metal, but oscillates on a wavelength scale $\lambda_{F}$ (i.e. $\lambda_{F}==/ Q_{F}$ ).

The oscillation of the pairing wave function in the $\mathrm{F}$ metal is a reason for the oscillatory $\mathrm{S} / \mathrm{F}$ proximity effect, mentioned above, yielding a nonmonotonous, oscillating dependence of the superconducting critical temperature $T_{c}$ on the ferromagnetic layer thickness $d_{F}[11,12]$. The phenomenon is explained by changing the interference conditions periodically between constructive and destructive upon changing the thickness of the ferromagnetic film thickness. Then, the flux of the pairing function through the interface of incidence is modulated. As a result, the coupling between the $\mathrm{S}$ and $\mathrm{F}$ layers is periodically modulated with increasing $d_{F}$, and the superconducting $T_{c}$ oscillates as a function of $d_{F}$. The amplitude of the $T_{c}$ oscillation depends sensitively on the superconducting layer thickness. It is obvious enough that for thick superconducting layer, $d_{F}>>\xi_{S}$ ( $\xi_{S}$ is the superconducting coherence length), the outer surface of the $\mathrm{S}$ layer, which determines actual $T_{c}$ of the system, is insensitive to the interface perturbation from the opposite side.

Recently, these mesoscopic $T_{c}$ oscillations in $\mathrm{S} / \mathrm{F}$ bilayers were realized in a series of $\mathrm{Nb} / \mathrm{Cu}_{1-x} \mathrm{Ni}_{x}$ bilayers with variable $\mathrm{Cu}_{1-x} \mathrm{Ni}_{x}$ ferromagnetic alloy thickness prepared with a technique described below (see detailed descriptions in Refs. 13, 14). Not only oscillatory, but pronounced reentrant, i.e., first suppressed and then recovered superconductivity as a function of $d_{F}$ were observed in these experiments. These findings turned out to be available owing to the advanced deposition technology [15] yielding significant improvement of the superconducting properties of thin $\mathrm{Nb}$ films and $\mathrm{Nb} / \mathrm{Cu}_{1-x} \mathrm{Ni}_{x}$ nanostructures in general, compared with conventional deposition techniques.

For fabrication of the S/F bilayers, niobium was chosen as a superconducting material because of rather high $T_{c}$, and $\mathrm{Cu}_{1-x} \mathrm{Ni}_{x}(x=0.59)$ alloy as a ferromagnetic material. The choice of the alloy instead of a conventional elemental ferromagnet has the following advantages for the experimentalist. The oscillation length $\lambda_{F 0}=2 \pi \hbar v_{F} / E_{\text {ex }}$ in strong clean ferromagnets, like iron, nickel or cobalt, is extremely short, because the exchange splitting energy $E_{\text {ex }}$ is usually in the range $0.1-1.0 \mathrm{eV}$ [16-19]. Thus, to detect the oscillatory behavior of $T_{c}$ experimentally, the ferromagnetic layer thickness $d_{F}$ must be very small, e.g., between 0.6 to $2.5 \mathrm{~nm}$ for pure $\mathrm{Ni}$ [19]. Weak ferromagnets with an order of magnitude smaller exchange splitting of the conduction band allow to observe the effect at much larger thicknesses $d_{F}$ of about 5-25 $\mathrm{nm}$ which can be much easier controlled and characterized. Moreover, for the long-wavelength oscillations, the atomic-scale interface roughness has not any longer a dominating effect on the extinction of the interference patterns.

In the first kind of samples the superconducting layer was of variable thickness, $d_{\mathrm{Nb}} \approx 4-47 \mathrm{~nm}$, prepared by a routine utilizing a wedge deposition technique described in detail in $[13,14]$. The $\mathrm{Cu}_{1-x} \mathrm{Ni}_{x}$ layer was flat with a thickness fixed at a infinite value of $d_{\mathrm{CuNi}}=56 \mathrm{~nm}$ [14] compared with the coherence length (see figures below). To prevent samples from deterioration in an ambient atmosphere all samples were always capped by an amorphous silicon layer of about $10 \mathrm{~nm}$ of thickness which is insulating at low temperatures. This series of samples has an auxiliary purpose to find a range of the $\mathrm{Nb}$ layer thickness most sensitive to variations of the ferromagnetic layer thickness.

In the second kind of samples the superconducting $\mathrm{Nb}$ layer was flat with a thickness fixed at a selected value in the range 6-15 nm. A special deposition technique with moving magnetron provided high quality, flat in the lateral extent of about $8 \mathrm{~cm}$, niobium layers with superconducting $T_{c 0}$ of the stand-alone film as high as $5.5 \mathrm{~K}$ at $d_{\mathrm{Nb}} \approx 5.7 \mathrm{~nm}$. To prepare samples with variable thickness of the ferromagnetic alloy layer a wedge-shaped film was deposited utilizing the off-symmetry mounting of the long substrate and intrinsic spatial gradient of the deposition rate. This technique, combined with the extensive Rutherford backscattering characterization of the thick- 
ness profile of the wedge was successfully applied to obtain a series of samples by cutting the wedge into strips across the thickness gradient $[13,14]$. Thus, 36-40 samples were obtained with variable $\mathrm{Cu}_{1-x} \mathrm{Ni}_{x}$ layer thickness in the range $d_{\mathrm{CuNi}} \approx 1-40 \mathrm{~nm}$, prepared at identical conditions in a single deposition run.

The superconducting critical temperature $T_{c}$ was determined from the midpoints of the resistive transitions curves $R(T)$. The width of transitions $\left(0.1 R_{N}-0.9 R_{N}\right.$ criteria, where $R_{N}$ is the normal state resistance just above $T_{c}$ ) for most of the investigated samples was below $0.2 \mathrm{~K}$, thus allowing to determine the $T_{c}$ with a sufficient accuracy.

Figure 2 demonstrates the dependence of the superconducting transition temperature on the $\mathrm{Cu}_{41} \mathrm{Ni}_{59}$ layer thickness. The thickness of the flat $\mathrm{Nb}$ layer is fixed, $d_{\mathrm{Nb}} \approx 14.1 \mathrm{~nm}$ (S23 series), $d_{\mathrm{Nb}} \approx 7.8 \mathrm{~nm}$ (S22 series), $d_{\mathrm{Nb}} \approx 7.3 \mathrm{~nm}$ (S15 series) and $d_{\mathrm{Nb}} \approx 6.2 \mathrm{~nm}$ (S21 series). The transition temperature $T_{c}$ for the specimens with $d_{\mathrm{Nb}} \approx 14.1 \mathrm{~nm}$ reveals a nonmonotonous behavior with a shallow minimum at about $d_{\mathrm{CuNi}} \approx 7.0 \mathrm{~nm}$. For the thinner niobium layer $\left(d_{\mathrm{Nb}} \approx 7.8 \mathrm{~nm}\right)$, the transition temperature shows a pronounced minimum with subsequent increase of $T_{c}$ to above $2.5 \mathrm{~K}$. Further decrease of the niobium layer thickness $\left(d_{\mathrm{Nb}} \approx 7.3 \mathrm{~nm}\right)$ leads already to the reentrant behavior, when the transition temperature drops when increasing the ferromagnetic layer thickness till the superconductivity is completely suppressed. Upon successive increase of $d_{\mathrm{CuNi}}$ the superconductivity restores, and $T_{c}$ gets values as high as $2 \mathrm{~K}$. For the thinnest $\mathrm{Nb}$ layer $\left(d_{\mathrm{CuNi}} \approx 6.2 \mathrm{~nm}\right)$, the superconducting $T_{c}$ sharply drops upon increasing the ferromagnetic $\mathrm{Cu}_{41} \mathrm{Ni}_{59}$ layer thickness till at certain thickness $d_{\mathrm{CuNi}} \approx 2.5 \mathrm{~nm}$ the superconductivity is fully suppressed.

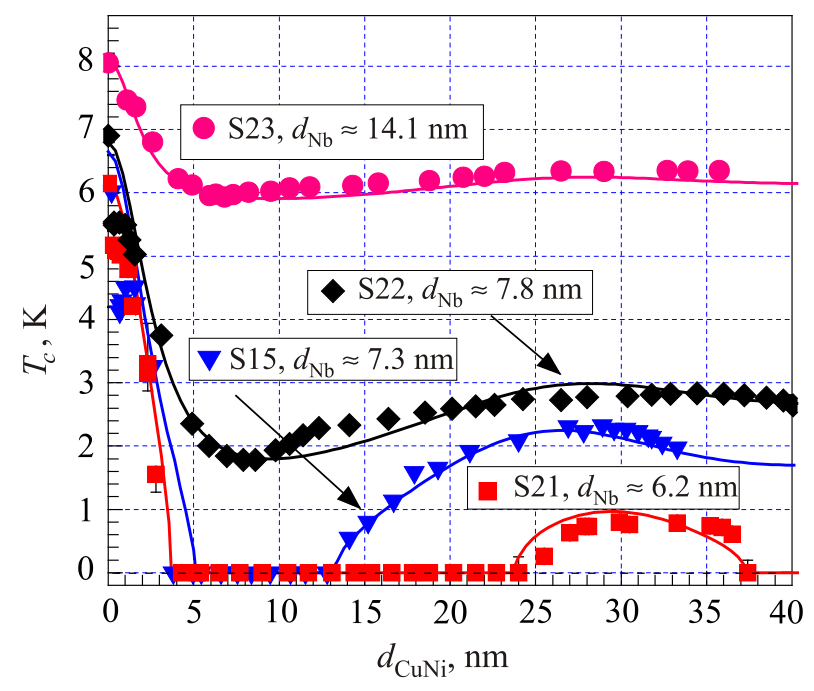

Fig. 2. Nonmonotonous $T_{c}\left(d_{F}\right)$ dependences for the $\mathrm{Nb} / \mathrm{Cu}_{1-x} \mathrm{Ni}_{x}$ bilayers $(x=0.59)$. Solid curves are calculated with values of parameters given in the text. The calculations give no further reentrance of superconductivity for the S21 sample series above $d_{\mathrm{CuNi}}>40 \mathrm{~nm}$.
Then, in the range $d_{\mathrm{CuNi}} \approx 2.5-24 \mathrm{~nm}$, the superconducting transition temperature vanishes $\left(T_{c}\right.$ is at least lower than the lowest temperature reached in our cryogenic setup, $40 \mathrm{mK}$ ). With a subsequent increase of the $\mathrm{Cu}_{1-x} \mathrm{Ni}_{x}$ layer thickness, superconductivity restores again at $d_{\mathrm{CuNi}} \approx 25.5 \mathrm{~nm}$, reaching a level of about $0.8 \mathrm{~K}$ at $d_{\mathrm{CuNi}} \approx 30 \mathrm{~nm}$, and then drops down again below $40 \mathrm{mK}$ at $d_{\mathrm{CuNi}} \approx 37.5 \mathrm{~nm}$. This phenomenon of a double suppression of superconductivity is the first experimental evidence for a multiple reentrant behavior of the superconducting state in $\mathrm{S} / \mathrm{F}$ layered systems [14].

The data simulation procedure includes coordinated fitting of the $T_{c}\left(d_{\mathrm{Nb}}\right)$ dependence, and $T_{c}\left(d_{\mathrm{CuNi}}\right)$ dependences from Fig. 2. The general fitting strategy is described in detail in the previous papers $[13,14,19]$. The solid curves in the figure show results of the calculations for the following set of parameters: curves S15, S21, S22, and S23; the stand-alone $\mathrm{Nb}$ film superconducting transition temperature $T_{c 0, \mathrm{Nb}}\left(d_{\mathrm{CuNi}}=0 \mathrm{~nm}\right)=6.67,6.2,6.85$, and $8.0 \mathrm{~K}$, respectively; the superconducting coherence length of $\mathrm{Nb}$, $\xi_{S}=6.3,6.5$, and $6.6 \mathrm{~nm}$; the ratio of the Sharvin conductances of the contacting metals, $N_{F} v_{F} / N_{S} v_{S}=0.22$ for all; the $\mathrm{Nb} / \mathrm{CuNi}$ interface transparency parameter $T_{F}=0.67,0.65,0.61$, and 0.44 ; the ratio of the conduction electron mean-free path to the coherence length in the CuNi alloy layer, $l_{F} / \xi_{F 0}=1.3,1.1,1.1$, and $1.1 ; \xi_{F 0}=9.5$, $11.2,10.7$, and $10.8 \mathrm{~nm}$. Although a common set of parameters has been used as a first step to fit the data, then, the superconducting coherence length, $\xi_{S}$, and the magnetic coherence length, $\xi_{F 0}$, were varied within a $5 \%$ range, and the $\mathrm{S} / \mathrm{F}$ interface transparency parameter $T_{F} \in[0, \infty)$ was varied within the range $[0.43,0.65]$ to obtain better fits for the individual curves. These degrees of freedom that were allowed for the physical parameters are well within the scatter which can be expected from variations of the deposition conditions from run to run.

The electron mean-free-path $l_{F} \approx 11.8-12.4 \mathrm{~nm}$, used in the above calculations, appeared to be longer than the coherence length $\xi_{F 0}=9.5-11.2 \mathrm{~nm}$. According to Ref. 20, $l_{F} \approx 4.4 \mathrm{~nm}$ for a $\mathrm{Cu}_{47} \mathrm{Ni}_{53}$ alloy with resistivity $\rho_{F}=57 \mu \Omega \cdot \mathrm{cm}$ (bulk material, $T=10 \mathrm{~K}$ ). Assuming that the product $\left\langle\rho_{F} l_{F}\right\rangle \approx 2.5 \cdot 10^{-5} \mu \Omega \cdot \mathrm{cm}^{2}$ remains unchanged upon adding impurities [21] one gets $l_{F} \sim 10 \mathrm{~nm}$ for our $\mathrm{Cu}_{41} \mathrm{Ni}_{59}$ alloy using the data for the low-temperature resistivity from Ref. $13, \rho_{F} \approx 25 \mu \Omega \cdot \mathrm{cm}$. Thus, both the proximity and the resistivity analysis indicate that the $\mathrm{Cu}_{41} \mathrm{Ni}_{59}$ alloy is in between the "dirty" $\left(l_{F}<<\xi_{F 0}\right)$ and the "clean" $\left(l_{F}>>\xi_{F 0}\right)$ cases.

Using material parameters obtained from the nonmonotonous and reentrant superconductivity behavior described above, it is possible to plot $T_{c}\left(d_{F}\right)$ curves of a $\mathrm{F} / \mathrm{S} / \mathrm{F}$ spin-switch core structure [22-25] and estimate $\Delta T_{c}=T_{c}^{A P}-T_{c}^{P}$, where the AP superscript stands for the antiparallel alignment, and $\mathrm{P}$ for the parallel alignment of magnetizations in the $\mathrm{F} / \mathrm{S} / \mathrm{F}$ trilayer. 


\section{The $F / S / F$ core structure of superconducting spin valve}

In this section we give derivation of the main expressions for calculating the superconducting $T_{c}$ of a $\mathrm{F} / \mathrm{S} / \mathrm{F}$ spin-valve core structure with physical parameters obtained from experiments on $\mathrm{S} / \mathrm{F}$ bilayers (this section is based on the original version from Ref. 14). The formulas for $T_{c}$ of $\mathrm{S} / \mathrm{F}$ bilayers used for calculations of the curves in Fig. 2 follow as a particular case of the $\mathrm{F} / \mathrm{S} / \mathrm{F}$ structure with parallel alignment of the F-layer magnetizations and twice thicker the $\mathrm{S}$ layer compared with those in bilayers.

First of all, let us note that our estimations made from the proximity effect as well as the resistivity data indicate that the coherence length $\xi_{F 0}$ and the conduction electron mean-free path $l_{F}$ are of the same order, $\xi_{F 0} \sim l_{F} \sim 10 \mathrm{~nm}$. Thus, the samples refer to the intermediate case in between of the dirty and the clean cases. Strictly speaking, the dirtycase theory based on the Usadel equations [26] is valid at the condition $l_{F}<<\xi_{F 0}$ which is clearly not fulfilled in our samples. Then, the Eilenberger theory [27], reformulated for S/F hybrids [28-30], comes into play. The advantage of the Eilenberger formulation is that it can be applied for arbitrary electron mean-free path, however, the equations are anisotropic and hard to solve analytically in the general case. A solution for the strong and clean ferromagnet $\left(\xi_{F 0}<<l_{F}\right)$ was presented in [28], while analysis for the weak proximity-effect regime (low-transparent $\mathrm{S} / \mathrm{F}$ interface) and arbitrary $\xi_{F 0}$ and $l_{F}$ was given in [30].

The solution to the problem was proposed in Ref. 11, where the anisotropic Eilenberger kernel was numerically averaged over trajectories, and the resulting oscillating decay of the average kernel was fitted with a single mode of the complex wave vector, varying the ratio $l_{F} / \xi_{F 0}$ in the range $0.5-5$ (see pages $155-156$ in Ref. 11). It appeared that the wave vector of this mode fits the Usadel's solution wave vector with the replacement of $D_{F}=l_{F} v_{F} / 3$ by $D_{F}=v_{F} l_{F} /\left(1+i l_{F} / \xi_{F 0}\right)$. The similar result was obtained by Linder et al. [30] (though for the one-dimensional transmissions) when they considered the dirty limit of their general solutions (see Eqs. (22) and (23) of Ref. 30). Important to mention that the single decay length that appears in the extended Usadel approach includes approximately all types of electron scatterings, the spin-reversal scatterings too.

For simplification, we took a $\mathrm{F}_{L} / \mathrm{S} / \mathrm{F}_{R}$ trilayer design with the S-layer thickness $d_{S}=2 \tilde{d}_{S}$, because the spinvalve core structure can be considered as a stack of two bilayers $\mathrm{F} / \tilde{\mathrm{S}}$ and $\tilde{\mathrm{S}} / \mathrm{F}$, building a $\mathrm{F} / 2 \tilde{\mathrm{S}} / \mathrm{F}$ trilayer with the left F-layer thickness $d_{F L}$, and the right F-layer thickness $d_{F R}$. The magnetization directions of the ferromagnetic $\mathrm{F}_{L}$ and $\mathrm{F}_{R}$ layers could be either parallel ( $\mathrm{P}$ alignment) or antiparallel (AP alignment). The other parameters: the layers thickness and transparencies of the $\mathrm{F}_{L} / \mathrm{S}$ and $\mathrm{S} / \mathrm{F}_{R}$ interfac- es can be varied. This is possible while the growth conditions for the first ferromagnetic layer (i.e., $\mathrm{F}_{L}$ on the substrate) and the second one ( $\mathrm{F}_{R}$ deposited on the $\mathrm{S}$ layer) are essentially different. Using our advanced wedge deposition technique, patented and described in $[13,14,19]$ we can exclude the influence of possible variation of the deposition conditions, preparing the ferromagnetic layers as wedges, and searching for the optimal specimen after cutting the long wedge-shape sample across the thickness gradient.

A similar nonsymmetrical design was fabricated and examined by Fauré et al. [31] (and also by Cadden-Zimansky et al. [32] with different F-layer thickness), however, in that case they considered a thin superconducting layer, $2 \tilde{d}_{S} \leq \xi_{S}$ (in the notations of paper [31] the superconducting layer thickness is determined as $d_{S} \leq \xi_{S}$ ). In our case the analysis is valid also at the condition $2 \tilde{d}_{S} \leq \xi_{S}$, as shown from the experimental data on bilayers.

To solve the problem of finding the critical temperature $T_{c}$ (either $T_{c}^{P}$ for the $\mathrm{P}$ alignment, or $T_{c}^{A P}$ for the AP alignment) the linearized Usadel equations had been solved $[9,26]$ for the pairing function $\Phi(x, \omega>0)$ :

$$
\left\{\omega+i \frac{E_{\mathrm{ex}}}{2}-\frac{1}{2} D_{F} \frac{d^{2}}{d x^{2}}\right\} \Phi_{F}(x, \omega)=0
$$

for each of the $\mathrm{F}$ layers, and

$$
\left\{\omega-\frac{1}{2} D_{S} \frac{d^{2}}{d x^{2}}\right\} \Phi_{S}(x, \omega)=\Delta(x)
$$

for the $\mathrm{S}$ layer, where $\Delta(x)$ is the superconducting order parameter, $D_{S}\left(D_{F}\right)$ is the diffusion coefficient of electrons in the $\mathrm{S}(\mathrm{F})$ layer, $E_{\mathrm{ex}}$ is the exchange splitting of the conduction band, and $\omega=\pi T(2 n+1)$ is the Matsubara frequency. Here, we set $\hbar$ and $k_{B}$ equal to one. The solutions have to satisfy the boundary conditions [11,33-35],

$$
\frac{d}{d x} \Phi_{F}\left( \pm \tilde{d}_{S} \pm d_{\frac{F R}{F L}}, \omega\right)=0
$$

at the outer surfaces, and

$$
\begin{gathered}
N_{S} D_{S} \frac{d}{d x} \Phi_{S}=N_{F} D_{F} \frac{d}{d x} \Phi_{F}, \\
\mp D_{F}\left(\mathbf{n}_{F} \nabla_{x} \Phi_{F}\right)=\frac{v_{F} T_{F}}{2}\left(\Phi_{S}-\Phi_{F}\right)
\end{gathered}
$$

at the interfaces of the superconductor with the ferromagnets. In Eqs. (3)-(5), $N_{S}\left(N_{F}\right)$ are the electronic density of states of the $\mathrm{S}(\mathrm{F})$ layer, $\mathbf{n}_{F}$ is a vector of the outward unit normal to the right $(-)$ or left $(+)$ S/F interface, $T_{F}$ is the dimensionless interface transparency parameter, $T_{F} \in[0, \infty)[11,34], v_{F}$ is the Fermi velocity of the ferromagnetic alloy, and $x$ is the space coordinate. 
The reduced critical temperature of the $\mathrm{S} / \mathrm{F}$ sample, $t_{c}=T_{c} / T_{c 0}$ in the single-mode approximation was found by solving the equation

$$
\ln t_{c}=\Psi\left(\frac{1}{2}\right)-\operatorname{Re} \Psi\left(\frac{1}{2}+\frac{\phi^{2}}{2 t_{c}\left(\tilde{d}_{S} / \xi_{S}\right)^{2}}\right),
$$

where $\phi=k_{S} \tilde{d}_{S}$, and $k_{S}$ is the propagation momentum of the pairing function in the $\mathrm{S}$ layer, $\phi=\phi^{P}$ for the $\mathrm{P}$ alignment of magnetizations, and $\phi=\phi^{A P}$ for the AP alignment of magnetizations, $\xi_{S}=\left(D_{S} / 2 \pi T_{c 0}\right)^{1 / 2}$ is the superconducting coherence length in the $\mathrm{S}$ layer, $T_{c 0}$ is the critical temperature of the stand-alone superconducting layer.

Now we consider in detail the symmetric spin-valve core structure with equivalent physical parameters of the ferromagnetic layers and interfaces. Then, for the $\mathrm{P}$ alignment of magnetizations we find the equation for $\phi=\phi^{P}$ :

$$
\phi^{P} \tan \phi^{P}-R=0,
$$

and for the AP alignment we get

$$
\left(\phi^{A P} \tan \phi^{A P}-R^{\prime}\right)\left(R^{\prime} \tan \phi^{A P}+\phi^{A P}\right)+\left(R^{\prime \prime}\right)^{2} \tan \phi^{A P}=0,
$$

where

$$
R=\frac{N_{F} D_{F}}{N_{S} D_{S}} \frac{k_{F} d_{S} \tanh \left(k_{F} d_{F}\right)}{1+\frac{2 D_{F} k_{F}}{T_{F} v_{F}} \tanh \left(k_{F} d_{F}\right)}, \quad k_{F} d_{F}=\sqrt{\frac{i E_{\mathrm{ex}}}{D_{F}}} d_{F},
$$

and $R^{\prime}$ and $R^{\prime \prime}$ are the real and imaginary parts of the $R$ given above, $N_{S(F)}$ is the electron density of states at the Fermi energy of superconductor (ferromagnet). For more details see Ref. 14. When inserted into Eq. (6) the solution of Eq. (7) for $\phi^{P}$ gives the critical temperature $T_{c}^{P}$ of the symmetric $\mathrm{F} / \mathrm{S} / \mathrm{F}$ structure for the $\mathrm{P}$ alignment of magnetizations. The same is true if we substitute the solution of Eq. (8) for $\phi^{A P}$ into Eq. (6) to get the critical temperature $T_{c}^{A P}$ in the symmetric $\mathrm{F} / \mathrm{S} / \mathrm{F}$ structure for the antiparallel alignment of magnetizations of the $\mathrm{F}$ layers.

Taking the values of the physical parameters from the fitting of $T_{c}\left(d_{F}\right)$ and $T_{c}\left(d_{S}\right)$ for the S/F bilayers we can calculate the magnitude of the spin-valve effect expected for the symmetric F/S/F core structure. In Fig. 3 are shown the results of the $T_{c}\left(d_{F}\right)$ calculations for the symmetric $\mathrm{F} / \mathrm{S} / \mathrm{F}$ structure at $\mathrm{P}$ and $\mathrm{AP}$ alignment of magnetizations of the ferromagnetic layers. That calculations were done for the following set of parameters: $d_{S}=d_{\mathrm{Nb}}=11.8 \mathrm{~nm}$ (a), $d_{S}=d_{\mathrm{Nb}}=13.9 \mathrm{~nm}(\mathrm{~b}) ; T_{c 0, \mathrm{Nb}}\left(d_{\mathrm{CuNi}}=0 \mathrm{~nm}\right)=7.7$ (a), $7.9 \mathrm{~K}$ (b); taken in accordance with Fig. 2, and in both cases $\xi_{S}=6.6 \mathrm{~nm} ; N_{F} v_{F} / N_{S} v_{S}=0.22 ; T_{F}=0.6 ; l_{F} / \xi_{F 0}=1.1$; $\xi_{F 0}=10.5 \mathrm{~nm}$.

The estimations show that for the Nb-layer thickness in the range, $d_{\mathrm{Nb}}=12.0-15.0 \mathrm{~nm}, \Delta T_{c}$ can be rather high, as

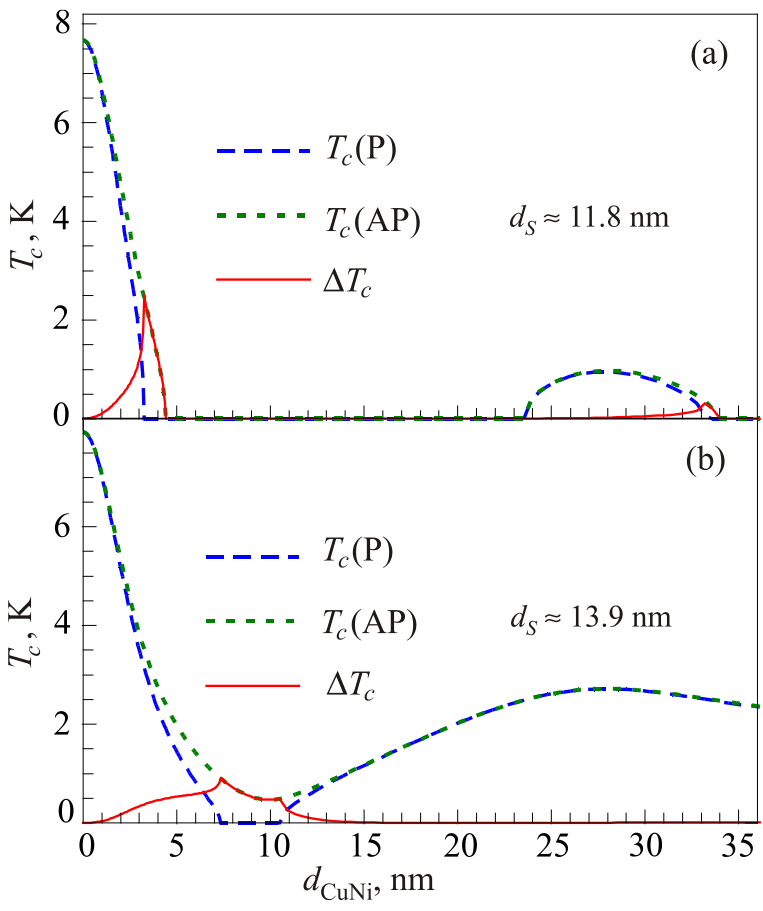

Fig. 3. The $T_{c}\left(d_{F}\right)$ curves of the superconducting F/S/F spin-valve core structure with $d_{S}=d_{\mathrm{Nb}}=11.8 \mathrm{~nm}(\mathrm{a}) ; d_{S}=d_{\mathrm{Nb}}=13.9 \mathrm{~nm}$ (b); calculated using the set of parameters given in the text.

large as $2 \mathrm{~K}$. In Fig. 4 we plot the maximal change of the critical temperature, $\Delta T_{c}^{\max }=\max \left(T_{c}^{A P}-T_{c}^{P}\right)$ due to the change of the magnetization alignment of the CuNi layers from parallel to antiparallel, together with the related

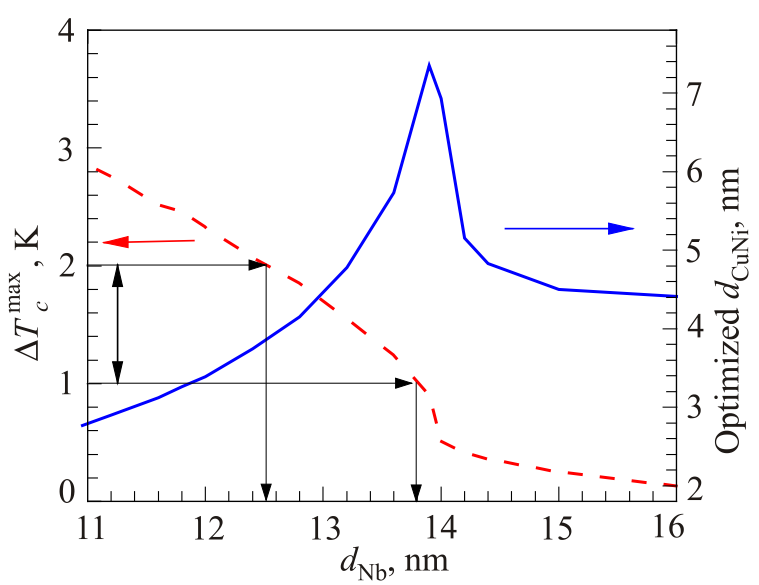

Fig. 4. The maximal difference of the critical temperatures for AP and $\mathrm{P}$ alignments of magnetizations in the symmetric $\mathrm{F} / \mathrm{S} / \mathrm{F}$ spinswitch core structure, $\Delta T_{c}^{\max }=\max \left(T_{c}^{A P}-T_{c}^{P}\right)$, versus Nb-layer thickness is shown by the dashed curve. The values of parameters for the calculations are the same as for Fig. 3. The thickness of the CuNi layers, at which an actual maximum is reached (see Fig. 3), is given by the solid curve. The Fig. 4 allows to select a range of the Nb-layer thickness $d_{\mathrm{Nb}}=12.5-13.8 \mathrm{~nm}$ (see thin vertical arrows), and the CuNi-layer thickness $d_{\mathrm{CuNi}}=3.5-5.0 \mathrm{~nm}$ (continue thin horizontal arrows) for which $\Delta T_{c}^{\max }$ is in the range $1-2 \mathrm{~K}$ (see thick double-head arrow). 
thickness $d_{\mathrm{CuNi}}$ as a function of the Nb-layer thickness. The value of $\Delta T_{c}^{\max }$ increases for decreasing $\mathrm{Nb}$-layer thickness. The related (i.e., "optimized") CuNi layer thickness passes through a maximum and then decreases. The shaded region is available for our deposition technique.

\section{Other possible design of superconducting spin valve - "triplet switching"}

Recently, an alternative F1/N/F2/S design of the superconducting spin valve [36] attracted much attention [37-39] because of possible high sensitivity of the superconducting $T_{c}$ to generation of the long-range triplet component of superconducting pairing $[40,41]$. Indeed, recent calculations have shown [42] that not only the "direct switching" $\left(T_{c}^{A P}>T_{c}^{P}\right)$ is possible, but also the "inverse switching" $\left(T_{c}^{P}>T_{c}^{A P}\right)$ can be realized [43]. Moreover, a novel "triplet switching" $\left(T_{c}^{\text {Triplet }}<\left\{T_{c}^{A P}>T_{c}^{P}\right\}\right)$ was predicted [42], which can be most spectacularly detected as a reentrant behavior of superconductivity as a function of the angle between magnetic moments of the F1 and F2 ferromagnetic layers. It has been shown that superconducting $T_{c}$ for the F1/N/F2/S structure, calculated with physical parameters derived from the experiments described above, keeps all features predicted in [42] for the simplified model, and the triplet component was detected in $\mathrm{F} 1 / \mathrm{N} / \mathrm{F} 2 / \mathrm{S}$ multilayer structure [44-48] opening the perspectives for the triplet spin-valve design for superconducting electronics [49,50]. One can see that the functionality of the superconductor/ferromagnet layered nanostructures can be successfully utilized to build a novel base elements for superconducting electronics development.

\section{Acknowledgments}

The author gratefully acknowledge a long-term cooperation with S. Gsell, S. Horn, J.-M. Kehrle, H.-A. Krug von Nidda, M.Yu. Kupriyanov, J. Lindner, C. Müller, J. Moosburger-Will, R. Morari, A. Prepelita, E. Antropov, E. Nold, G. Obermeier, V. Oboznov, V.V. Ryazanov, M. Schreck, R. Tidecks, L.R. Tagirov, and V.I. Zdravkov. Special thanks to A. v. Humboldt Foundation (KarlsruheINT/Kishinev-IIEN Institutspartnerschaften-Project), to STCU project N5982 "Experimental investigation of the proximity effect in layered superconductor/ferromagnet hybrid structures", and STCU project N6329 for the partial support of the work.

1. V.L. Ginzburg, Zh. Eksp. Teor. Fiz. 31, 202 (1956) [Sov. Phys. JETP 4, 153 (1957)]

2. M.B. Maple and Ø. Fischer, Superconductivity in Ternary Compounds, Springer-Verlag (1982).

3. K.-H. Müller and V.N. Narozhnyi, Rep. Prog. Phys. 64, 943 (2001).

4. V.P.S. Awana, in Frontiers in Magnetic Materials, Springer (2005), p. 531.
5. Z. Radović, L. Dobrosavljević-Grujić, A.I. Buzdin, and J. Clem, Phys. Rev. B 38, 2388 (1988).

6. E.A. Demler, G.B. Arnold, and M.R. Beasley, Phys. Rev. B 55, 15174 (1997).

7. A.A. Golubov, M.Yu. Kupriyanov, and E. Il'ichev, Rev. Mod. Phys. 76, 411 (2004).

8. I.F. Lyuksyutov and V.L. Pokrovsky, $A d v$. Phys. 54, 67 (2005).

9. A.I. Buzdin, Rev. Mod. Phys. 77, 935 (2005).

10. F.S. Bergeret, A.F. Volkov, and K.B. Efetov, Rev. Mod. Phys. 77, 1321 (2005).

11. L.R. Tagirov, Physica C 307, 145 (1998).

12. M.G. Khusainov and Yu.N. Proshin, Phys. Rev. B 56, 14283 (1997).

13. V.I. Zdravkov, A.S. Sidorenko, G. Obermeier, S. Gsell, M. Schreck, C. Müller, S. Horn, R. Tidecks, and L.R. Tagirov, Phys. Rev. Lett. 97, 057004 (2006).

14. V.I. Zdravkov, J. Kehrle, G. Obermeier, S. Gsell, M. Schreck, C. Müller, H.-A. Krug von Nidda, J. Lindner, J. MoosburgerWill, E. Nold, R. Morari, V.V. Ryazanov, A.S. Sidorenko, S. Horn, R. Tidecks, and L.R. Tagirov, Phys. Rev. B 82, 054517 (2010).

15. V. Zdravkov, R. Morari, Dispozitiv de obtinere a peliculelor supraconductoare (Device for preparation of superconducting films). Authors: A. Sidorenko, Patent of the Republic of Moldova No. 175(134), published 31.03.2010.

16. J.S. Jiang, D. Davidović, D.H. Reich, and C.L. Chien, Phys. Rev. Lett. 74, 314 (1995).

17. Th. Mühge, N.N. Garif'yanov, Yu.V. Goryunov, G.G. Khaliullin, L.R. Tagirov, K. Westerholt, I.A. Garifullin, and H. Zabel, Phys. Rev. Lett. 77, 1857 (1996).

18. I.A. Garifullin, D.A. Tikhonov, N.N. Garif'yanov, L. Lazar, Yu.V. Goryunov, S.Ya. Khlebnikov, L.R. Tagirov, K. Westerholt, and H. Zabel, Phys. Rev. B 66, 020505 (2002).

19. A.S. Sidorenko, V.I. Zdravkov, A. Prepelitsa, C. Helbig, Y. Luo, S. Gsell, M. Schreck, S. Klimm, S. Horn, L.R. Tagirov, and R. Tidecks, Ann. Phys. (Berlin) 12, 37 (2003).

20. J. Kim, J. H. Kwon, K. Char, H. Doh, and H.-Y. Choi, Phys. Rev. B 72, 014518 (2005).

21. R.A. French, Cryogenics 8, 301 (1968).

22. L.R. Tagirov, Phys. Rev. Lett. 83, 2058 (1999).

23. A.I. Buzdin, A.V. Vedyayev, and N.V. Ryzhanova, Europhys. Lett. 48, 686 (1999).

24. I. Baladié, A. Buzdin, N. Ryzhanova, and A. Vedyayev, Phys. Rev. B 63, 054518 (2001).

25. Ya.V. Fominov, A.A. Golubov, and M.Yu. Kupriyanov, Pis'ma v ZhETF 77, 609 (2003) [JETP Lett. 77, 510 (2003)].

26. K.D. Usadel, Phys. Rev. Lett. 25, 507 (1970).

27. G. Eilenberger, Z. Phys. 214, 195 (1968).

28. B.P. Vodopyanov and L.R. Tagirov, Pis'ma v ZhETF 78, 1043 (2003) [JETP Lett. 78, 555 (2003)].

29. F.S. Bergeret, A.F. Volkov, and K.B. Efetov, Phys. Rev. B 64, 134506 (2002).

30. J. Linder, M. Zareyan, and A. Sudbø, Phys. Rev. B 79, 064514 (2009). 
31. M. Fauré, A. I. Buzdin, and D. Gusakova, Physica C 454, 61 (2007).

32. P. Cadden-Zimansky, Ya.B. Bazaliy, L.M. Litvak, J.S. Jiang, J. Pearson, J.Y. Gu, C.-Y. You, M.R. Beasley, and S.D. Bader, Phys. Rev. B 77, 184501 (2008).

33. M.Yu. Kupriyanov and V.F. Lukichev, Zh. Eksp. Teor. Fiz. 94, 139 (1988) [Sov. Phys. JETP 67, 1163 (1988)].

34. J. Aarts, J.M.E. Geers, E. Brück, A.A. Golubov, and R. Coehoorn, Phys. Rev. B 56, 2779 (1997).

35. L.R. Tagirov and N. García, Superlatt. and Microstruct. 41, 152 (2007).

36. S. Oh, D. Youm, and M.R. Beasly, Appl. Phys. Lett. 71, 2376 (1997).

37. K. Westerholt, D. Sprungmann, H. Zabel, R. Brucas, B. Hjörvarsson, D.A. Tikhonov, and I.A. Garifullin, Phys. Rev. Lett. 95, 097003 (2005).

38. G. Nowak, H. Zabel, and K. Westerholt, I. Garifullin, M. Marcellini, A. Liebig, and B. Hjörvarsson, Phys. Rev. B 78, 134520 (2008).

39. P.V. Leksin, N.N. Garif'yanov, I.A. Garifullin, J. Schumann, H. Vinzelberg, V.E. Kataev, R. Klingeler, O.G. Schmidt, and B. Büchner, Appl. Phys. Lett. 97, 102505 (2010).

40. F.S. Bergeret, A.F. Volkov, and K.B. Efetov, Phys. Rev. Lett. 64, 134506 (2001).

41. A.F. Volkov, F.S. Bergeret, and K.B. Efetov, Phys. Rev. Lett. 90, 117006 (2003).

42. Ya.V. Fominov, A.A. Golubov, T.Yu. Karminskaya, M.Yu. Kupriyanov, R.G. Deminov, and L.R. Tagirov, Pis'ma $v$ ZhETF 91, 329 (2010) [JETP Lett. 91, 308 (2010)].
43. P.V. Leksin, N.N. Garif'yanov, I.A. Garifullin, J. Schumann, V.E. Kataev, O.G. Schmidt, and B. Büchner, Phys. Rev. Lett. 106, 067005 (2011).

44. P.V. Leksin, N.N. Garif'yanov, I.A. Garifullin, Ya.V. Fominov, J. Schumann, Y. Krupskaya, V.E. Kataev, O.G. Schmidt, and B. Büchner, Phys. Rev. Lett. 109, 057005 (2012).

45. V.I. Zdravkov, J. Kehrle, G. Obermeier, D. Lenk, H.-A. Krug von Nidda, C. Müller, M.Yu. Kupriyanov, A.S. Sidorenko, S. Horn, R. Tidecks, and L.R. Tagirov, Phys. Rev. B 87, 144507 (2013).

46. A. Singh, S. Voltan, K. Lahabi, and J. Aarts, Phys. Rev. X 5, 021019 (2015).

47. P.V. Leksin, N.N. Garif'yanov, A.A. Kamashev, Ya.V. Fominov, J. Schumann, C. Hess, V.E. Kataev, B. Büchner, and I.A. Garifullin, Phys. Rev. B 91, 214508 (2015).

48. D. Lenk, V. Zdravkov, J.-M. Kehrle, G. Obermeier, A. Ullrich, R. Morari, H.-A. Krug von Nidda, C. Müller, M.Yu. Kupriyanov, A. Sidorenko, S. Horn, R. Deminov, L.R. Tagirov, and R. Tidecks, Beilstein J. Nanotechnology 7, 957 (2016).

49. A. Sidorenko, Fundamentals of Superconducting Nanoelectronics, Springer (2011).

50. Jacob Linder and J.W.A. Robinson, Nature Phys. 11, 307 (2015). 